\title{
The effect of craniectomy size on mortality, outcome, and complications after decompressive craniectomy at a rural trauma center
}

\author{
Cara L. Sedney, Terrence Julien ${ }^{1}$, Jacinto Manon², Alison Wilson³ \\ Department of Neurosurgery, West Virginia University, ${ }^{1}$ Department of Neuroscience, Marshall University, ${ }^{2}$ Penrose Centura Hospital, \\ Memorial Hospital, Colorado Springs, Colorado, USA, ${ }^{3}$ Department of Surgery, West Virginia University
}

\begin{abstract}
Introduction: Decompressive craniectomy (DC) has increasing support with current studies suggesting an improvement in both survival rates and outcomes with this intervention. However, questions surround this procedure; specifically, no evidence has indicated the optimal craniectomy size. Larger craniectomy is thought to better decrease intracranial pressure, but with a possible increase in complication rates. Our hypothesis is that a larger craniectomy may improve mortality and outcome, but may increase complication rates. Materials and Methods: A retrospective observational therapeutic study was undertaken to determine if craniectomy size is related to complication rates, mortality, or outcome. Our institution's Trauma Registry was searched for patients undergoing DC. Craniectomy size was measured by antero-posterior (AP) diameter. Mortality, outcome (through admission and discharge Glasgow Coma Score and Glasgow Outcome Scale), and complications (such as re-bleeding, re-operation, hygroma, hydrocephalus, infection, and syndrome of the trephined) were noted. Complications, mortality, and outcome were then compared to craniectomy size, to determine if any relation existed to support our hypothesis. Results: 20 patients met criteria for inclusion in this study. Craniectomy size as measured by AP diameter was correlated with a statistically significant improvement in mortality within the group. All patients with a craniectomy size less than $10 \mathrm{~cm}$ died. However, outcome was not significantly related to craniectomy size in the group. Similarly, complication rates did not differ significantly compared to craniectomy size. Discussion: This study provides Level 3 evidence that craniectomy size may be significantly related to improved mortality within our group, supporting our initial hypothesis; however, no significant improvement in outcome was seen. Similarly, in contrast to our hypothesis, complication rates did not significantly correlate with craniectomy size.
\end{abstract}

Key words: Craniectomy, neurotrauma, traumatic brain injury

\section{Introduction}

Decompressive craniectomy (DC) is a salvage procedure for patients with severe traumatic brain injury (TBI) or other conditions producing refractory increased intracranial pressure. The rationale for its use is based upon the Monro-Kellie doctrine and the procedure was first described by Kocher in 1901 and Cushing in 1905. ${ }^{[1]}$

\begin{tabular}{|l|l|}
\hline \multicolumn{2}{|c|}{ Access this article online } \\
\hline Quick Response Code: & Website: \\
\hline & www.ruralneuropractice.com \\
\cline { 2 - 3 } & \\
\hline
\end{tabular}

Initial experience with this procedure for traumatic injury was not encouraging; however, recent studies have shown a good neurologic outcome among survivors, defined as a Glasgow Outcome Scale (GOS) of 4-5, to be up to 50-70\%. ${ }^{[2]}$ Enthusiasm has grown for the use of this procedure at such a rate that in 2010 alone, over 130 publications were added to the literature. Although there is a marked heterogeneity of cerebral pathophysiologic processes among patients with post-traumatic brain swelling, ${ }^{[3]}$ decompressive craniectomy after traumatic brain injury can lead to a variety of therapeutic effects: Enlargement of the intracranial space and intracranial volume with consequent restoration of perimesencephalic cisterns, decrease in midline shift, and improvement of cerebral compliance; ${ }^{[4]}$ reduction of intracranial pressure (ICP); ${ }^{[5]}$ an increase in cerebral blood flow and perfusion; ${ }^{[4]}$ and improvement of cerebrovascular

\section{Address for correspondence:}

Dr. Cara L. Sedney, Department of Neurosurgery, West Virginia University, Robert C. Byrd Health Sciences Center, PO Box 9183 , Morgantown, West Virginia - 26506, USA. E-mail: csedney@hsc.wvu.edu. 
regulation. ${ }^{[6]}$ A multitude of controversies remain regarding particular aspects of the surgery, including optimal patient populations, timing, and technique. Although the results of the recent DECRA trial showed worse outcomes among patients undergoing DC for diffuse cerebral edema compared to maximal medical therapy, the outcomes are thought to be different for DC combined with mass lesion removal. ${ }^{[7]}$ The location of the craniectomy may vary between unilateral or bilateral temporofrontoparietal or bifrontal. ${ }^{[8]}$ The hinge craniectomy method has been described..$^{[9]}$ Although it is generally accepted that DC is most effective with expansion duraplasty, there are a number of methods for this as well. ${ }^{[10,11]}$ The optimal amount of extension of the craniectomy into the middle cranial fossa is debated, as is the proximity of the craniectomy edge to the midline. ${ }^{[12]}$ In addition, the optimal size of the craniectomy, which can balance maximal decompression and optimize outcome without increasing the risk of complications, is still unknown. In this study we sought to look specifically at craniectomy size and its relation to outcomes, mortality, and complications. Our hypothesis was that an increased craniectomy size may lead to improved outcomes and mortality, but may also lead to more complications.

\section{Materials and Methods}

This was a retrospective, IRB-approved study conducted at a rural, Level One trauma center with a large rural catchment area and predominantly blunt trauma mechanisms. The Trauma Registry was utilized to identify all patients who had a decompressive craniectomy for severe traumatic brain swelling and evidence of elevated intracranial pressure during the years 2002-2009. Exclusion criteria included age less than 18, patients who had DC for a reason other than trauma, death prior to the first post operative computed tomography (CT) scan (due to inability to measure craniectomy size), and those in whom a bilateral DC was performed as the initial surgery. Twenty patients met such criteria. Specific patient data included the mechanism of injury, admission and discharge Glasgow Coma Scale (GCS), Injury Severity Score, disposition upon discharge, and mortality. Clinical information from the discharge summary was used to confirm GCS and also formulate a discharge GOS. These factors were used to determine the overall outcome and mortality. The initial operative plan including craniectomy versus traditional craniotomy, as well as use of expansion duraplasty, was extracted from the operative note. Complications such as hydrocephalus, infection, stroke, hemorrhage, and reoperation were identified in order to relate these to the craniectomy size. Clinic documentation was utilized to identify long-term neurologic deficits such as monoparesis or seizures. When these were associated with the presence of midline shift on imaging and reversed with cranioplasty, these deficits were considered to constitute a "syndrome of the trephined." In order to assess the craniectomy size, each slice of the first postoperative CT scan was examined, with the largest anterior to posterior (AP) diameter of craniectomy recorded. Further CT scans were examined for evidence of hemorrhage, infarction, negative pressure mass effect, and subdural hygromas, as a further confirmation of complications. An attempt at calculating area of the craniectomy was made, using the previously published equation of De Bonis, adapted from that of Munch. ${ }^{[12,13]}$ [Figure 1]. However, upon use of the equation it appeared to be affected by the amount of cerebral swelling and herniation through the defect, raising the concern that this additional variable may be included as a confounder to the equation. Therefore, only AP diameter was used. The AP diameter was compared to mortality, outcome, and complications. With the assistance of a biostatistician, logistic regression analysis was used to assess the dependence of these factors on craniectomy size. A $P$ value of less than 0.05 was considered significant. Analyses were carried out using JMP Software (Version 9, SAS Institute, Cary, NC).

\section{Results}

Demographic information, which does not directly relate to the hypothesis, however may impact the applicability of our findings to other patient populations, is as follows: The patients in our study ranged in age from 18 to 66, with a mean age of 37.9. Eighteen of the

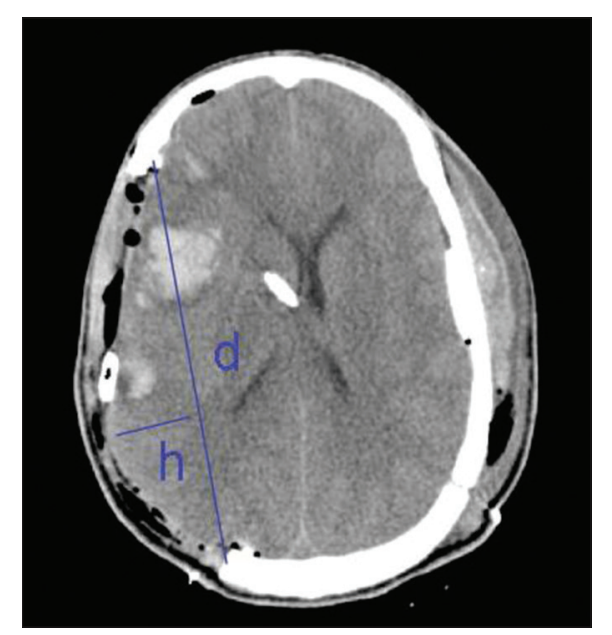

Figure 1: Method of craniectomy size measurement. D: Antero-posterior diameter as per De Bonis equation. H: Maximum perpendicular width from $d$ to margin of brain tissue 
patients were males. All patients had an associated mass lesion. Admission GCS ranged from 3 to 11, with a mean of 3.75; however, this may have been confounded by rapid-sequence intubation in the majority of patients. The most common mechanism of trauma was a motor vehicle accident in eight patients, followed by four falls, three motorcycle accidents, one pedestrian versus car injury, one assault, and one crush injury. Injury severity score (ISS) ranged from 16 to 45 , with an average of 31 . Individual data may be found in Table 1 . Initial CT result showed evidence of mass lesion with cerebral edema or mass effect in all patients. The decision to operate was made by patient exam and CT findings, as well as by intracranial pressure if a monitor was placed prior to surgery. All patients except four had a pre-operatively planned decompressive craniectomy. The remaining four had a craniectomy performed after cerebral edema was encountered in the operating room. All patients but one had an expansion duraplasty. In all cases the size of the craniectomy was dependent upon the discretion of the operating surgeon. The AP diameter of the craniectomy was found to range from 8.1 to $13.9 \mathrm{~cm}$.

The average diameter was $11.25 \mathrm{~cm}$. Craniectomy area as calculated by the De Bonis equation, although not used for analysis due to the confounding factor of brain herniation within the equation, averaged $124 \mathrm{~cm}^{2}$. A significant relationship $(P=0.0323)$ between increasing craniectomy size and decreased mortality was discovered. Furthermore, all patients with a craniectomy with AP diameter of less than $10 \mathrm{~cm}$ died. Outcome as measured by GOS and GCS upon discharge were not significantly related to craniectomy size; although there was a non-significant trend towards improved GCS and GOS with increased craniectomy size, this likely reflects mortality. The trend of improved GOS and GCS remained non-significant when the outcome of survivors alone was compared. The rate of each complication, as well as the complication rate in general, was not significantly related to craniectomy size. Sixteen patient experienced complications after surgery [Figure 2]. Two patients had hydrocephalus, defined as increasing ventricular size or intracranial pressure, or neurologic decline, requiring extra ventricular drain conversion to a ventriculoperitoneal shunt for permanent CSF diversion. Twelve patients had hypodensity on follow-up CT scan consistent with infarct. Four patients had neurologic deficit which resolved after cranioplasty consistent with syndrome of the trephined. Four patients required reoperation, three of which were for hemorrhage either on the ipsilateral or contralateral side. The remaining patient underwent surgery to increase the size of his craniectomy after persistent swelling and brain herniation. There were no operative site infections in our series. Ten patients had evidence of a subdural effusion on follow-up CT scans, but none of these were symptomatic. Since some complications such as hydrocephalus or syndrome of the trephined might be expected only with patients

Table 1: Patient clinical characteristic upon admission and at discharge

\begin{tabular}{|c|c|c|c|c|c|c|c|c|c|c|}
\hline Patient & Age & Sex & $\begin{array}{l}\text { Admit } \\
\text { GCS }\end{array}$ & ISS & Mechanism & APD & $\begin{array}{l}\text { Discharge } \\
\text { GCS }\end{array}$ & Dispo & GOS & Complications \\
\hline 1 & 65 & $\mathrm{~F}$ & 3 & 21 & Fall & 8.1 & 3 & Expired & 1 & None \\
\hline 2 & 60 & M & 3 & 29 & MCC & 7.7 & 3 & Expired & 1 & Infarct \\
\hline 3 & 66 & $\mathrm{~F}$ & 11 & 16 & MVC & 8.7 & 3 & Expired & 1 & None \\
\hline 4 & 42 & M & 7 & 21 & Fall & 9.6 & 3 & Expired & 1 & Infarct, hygroma, hemorrhage \\
\hline 5 & 20 & M & 3 & 30 & MVC & 10 & 7 & Home & 3 & Hydrocephalus, infarct, hemorrhage, reoperation \\
\hline 6 & 26 & M & 3 & 17 & ATV & 10.8 & 14 & Rehab facility & 4 & $\begin{array}{l}\text { Hydrocephalus, infarct, hygroma, hemorrhage, } \\
\text { reoperation }\end{array}$ \\
\hline 7 & 31 & M & 3 & 45 & ATV & 10.1 & 15 & Home & 5 & None \\
\hline 8 & 24 & M & 3 & 38 & MVC & 11.4 & $11 \mathrm{~T}$ & SNU & 3 & Syndrome of the trephined, hygroma \\
\hline 9 & 25 & M & 3 & 35 & MVC & 10.6 & 3 & Expired & 1 & Infarct, hemorrhage \\
\hline 10 & 19 & M & 3 & 26 & $\mathrm{MCC}$ & 11.5 & 15 & Home & 5 & Hygroma, hemorrhage \\
\hline 11 & 67 & M & 3 & 35 & MCC & 12.6 & $10 \mathrm{~T}$ & Acute Care Hospital & 3 & Hygroma \\
\hline 12 & 61 & M & 3 & 38 & Fall & 11.2 & $9 \mathrm{~T}$ & Nursing home & 3 & Syndrome of the trephined, hygroma \\
\hline 13 & 51 & M & 3 & 38 & Crush injury & 11.8 & 3 & Expired & 1 & None \\
\hline 14 & 23 & M & 3 & 26 & Ped vs car & 11.5 & 15 & Home & 5 & Infarct, hygroma \\
\hline 15 & 25 & M & 5 & 36 & MVC & 13 & $9 \mathrm{~T}$ & Rehab facility & 3 & Infarct, syndrome of the trphined, hygroma \\
\hline 16 & 18 & M & 3 & 45 & MVC & 13.1 & 15 & Rehab facility & 5 & Hemorrhage, reoperation \\
\hline 17 & 20 & M & 3 & 33 & Assault & 13.5 & $4 \mathrm{~T}$ & Acute Care Hospital & 2 & Infarct, hygroma \\
\hline 18 & 47 & M & 3 & 20 & Fall & 13.1 & 3 & Expired & 1 & Infarct, hemorrhage, reoperation \\
\hline 19 & 44 & M & 4 & 26 & MVC & 12.3 & 3 & Expired & 1 & Infarct \\
\hline 20 & 23 & $\mathrm{M}$ & 3 & 45 & MVC & 13.9 & 15 & Home & 5 & Infarct, syndrome of the trephined, hygroma \\
\hline
\end{tabular}



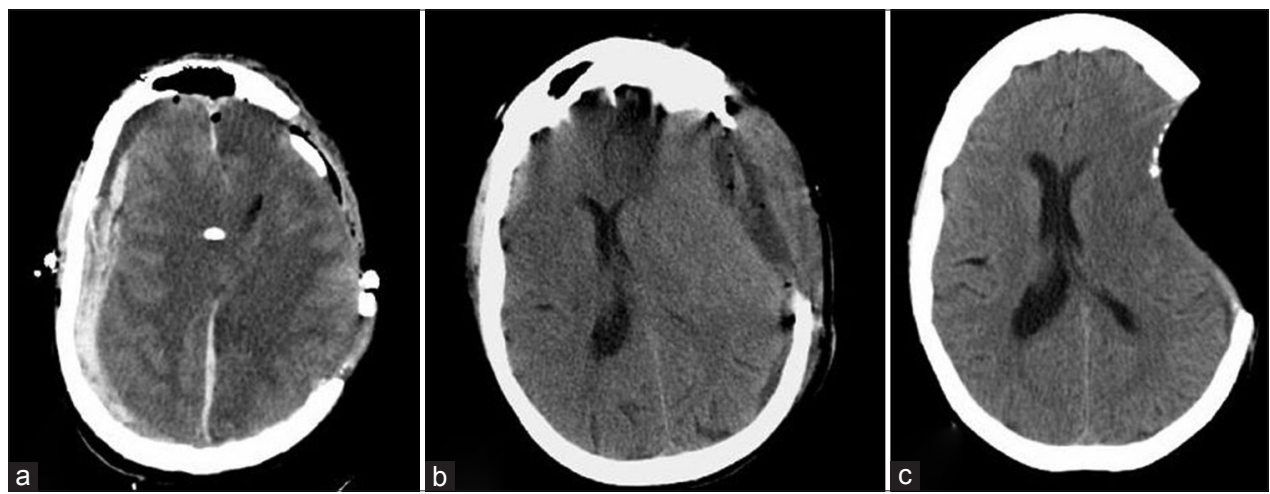

Figure 2: Post-operative complications after decompressive craniectomy. (a) Contralateral hemorrhage necessitating further surgery. (b) Asymptomatic subdural hygroma. (c) Sinking scalp flap which, accompanied by neurologic deterioration, signifies syndrome of the trephined

demonstrating long-term survival, complication rates were compared both overall and among survivors only. However, these factors remained non-significant when assessed only in survivors.

\section{Discussion}

This retrospective study examined the effect of craniectomy size on mortality, outcomes and complications, with the hypothesis that a larger craniectomy may improve mortality and outcome, but may also increase complications. We demonstrate a significant relationship of improved mortality with increasing craniectomy size in decompressive craniectomy for trauma and mass lesion evacuation, and our data suggests an absolute minimum AP diameter of $10 \mathrm{~cm}$. However, our data did not demonstrate a significant relationship between craniectomy size and outcome, nor complication rate. No change in outcome was seen, nor was there an increased rate of complications with increased craniectomy size. Both of these may relate to the sample size. The mortality rate, outcomes and complication rates are consistent with previously published data, with the exception of the number of patients who suffered from syndrome of the trephined. This is rarely mentioned in case series of DC, except in the form of case reports. Because of significant variation in the specifics of the craniectomy procedure, as well as geographical variation in its use, we feel that the experience of rural centers such as ours is valuable. The importance of craniectomy size has been recognized in the past, since a larger size increases the area of possible brain expansion during cerebral edema, while simultaneously decreasing the amount of venous congestion around the edges of the craniectomy site. However, increasing craniectomy size has been associated with increased complication rates, and DC is well known to be a morbid procedure with a reported overall complication rate of $55 \%$ in a recent retrospective review of a large series of such patients. ${ }^{[14]}$ Some of the earliest work on importance of craniectomy size was done in studies assessing DC in patients with malignant middle cerebral artery (MCA) infarction. Wirtz and colleagues assessed the area of craniectomy for malignant MCA infarction and found no relation to mortality for their study population although the average area of the craniectomy was only $84.3 \mathrm{~cm}^{2} \cdot{ }^{[15]}$ Wagner and colleagues looked at the AP diameter of the craniectomy and attempted to correlate this with outcome or mortality, but were unsuccessful. ${ }^{[16]}$ However, they noted that in smaller craniectomies, the area of infarcted brain was more likely to hemorrhage, and since this led to worse outcome among their study population, they postulated that a craniectomy smaller than $12 \mathrm{~cm}$ may lead to improved mortality or outcome by virtue of decreased incidence of hemorrhagic conversion of infarcted tissue. ${ }^{[16]}$ However, the difference in pathophysiology and patient populations in MCA infarction, as well as the stroke-specific complication of a hemorrhagic transformation of infarcted brain makes the applicability of this data to trauma patients uncertain. More recent studies in the TBI patient population have compared two extremes of craniectomy size, with a comparison of $8 \mathrm{~cm}$ and $12 \mathrm{~cm}$ diameter craniectomies in one study, and an additional one comparing $8 \mathrm{~cm}$ and $15 \mathrm{~cm}$ craniectomies. ${ }^{[17,18]}$ Although they found an improved outcome with the larger craniectomy size, an increased complication rate was also noted. In order to further elucidate the optimal craniectomy size, several other studies have been carried out. In 2000, Munch attempted to relate the area of the craniectomy with the amount of herniated brain, but found no relation of this to a decrease in midline shift or a decrease in ICP. ${ }^{[13]}$ In 2006, Skoglund et al. reported contradicting data showing a decrease in ICP depending upon the craniectomy size, but did not correlate this with outcome. ${ }^{[19]}$ In 2008, Gao and colleagues attempted to use biomechanical modeling to determine an optimal craniectomy size. ${ }^{[20]}$ Our results 
indicate that larger craniectomies may result in improved mortality for patients with severe brain injury with cerebral edema and an associated mass lesion. The $100 \%$ mortality of patients having a craniectomy less than $10 \mathrm{~cm}$ suggests an absolute minimum of craniectomy size. Furthermore, even when craniectomy is not planned preoperatively, a large craniotomy flap should be used in case severe swelling is encountered in the operating room. This may diminish the amount of axonal stretch or venous congestion of the herniated brain tissue. In spite of the difference in mortality, a difference in outcome based upon craniectomy size was not seen in our series. The rate of complications in DC is markedly higher than in traditional craniotomy. This is thought to be due both to the critical nature of illness and to the large craniectomy size. De Bonis has studied the incidence of hydrocephalus in craniectomy patients and postulates that both increasing craniectomy size and proximity of the superior craniectomy edge to midline increase the risk of hydrocephalus in this patient population. ${ }^{[12]}$ In our series, we did not demonstrate a higher complication rate with larger craniectomies although this may relate to the sample size. Our complication rates appear to be on par with other studies of DC and summarized by Honeybul, ${ }^{[21]}$ with the exception of four patients (20\%) who experienced the syndrome of the trephined, which has previously been reported to have a much lower incidence, only $1.2 \%$ in Honeybul's most recent large series. ${ }^{[14]}$ This may warrant further study. Overall, our findings further argue for larger craniectomy size, since survival appears to be improved without risk of increased complications. Central to the hypothesis of our paper is the method of calculating craniectomy size. Although not specifically assessed in this study, it appears that AP diameter may be superior to calculations of area to assess craniectomy size because of difficulty in differentiating craniectomy diameter and brain herniation. The craniectomy size has been measured by a number of methods in previous studies, both various measurements of area and AP diameter. The AP diameter, while a less direct measurement of craniectomy size than an area calculation, is reproducible and readily obtained. Several equations have been used for area calculation, but these are both overly complex and fail to account for the oval, rather than spherical, shape of the human skull. In addition, attempts at calculating the diameter of the craniectomy flap is often confounded by the diameter of the herniated brain tissue, introducing a confounder into the equation. This is especially critical since brain herniation likely is an indicator of more severe disease.

Enthusiasm for DC may falter given the recent results of the DECRA trial. ${ }^{[22]}$ Indeed, these results call for a careful assessment of benefits and risks for patients with severe traumatic brain injury, an intractable and costly problem worldwide with few therapeutic options. However, the craniectomy procedure is still in its infancy as regards to optimal patient populations, technique, and timing. Recent unpublished data by Narenthiran and colleagues have noted wide variation in practice regarding decompressive craniectomy among neurosurgeons worldwide. As noted by Aarabi and colleagues, DC combined with mass lesion evacuation rather than for diffuse cerebral edema constitute a distinct patient group. ${ }^{[7]}$ As noted by Servadei in his editorial regarding the DECRA study, DC combined with mass lesion evacuation is more common and more clinically applicable than that for diffuse cerebral edema. ${ }^{[23]}$ We hope that this retrospective review has helped add to the literature regarding specifics of the technique of decompressive craniectomy, and have demonstrated with this study that craniectomy size has a significant relationship with mortality, although we were unable to demonstrate a significant relationship with outcome or complication rate.

\section{References}

1. Olivecrona M, Rodling-Wahlstrom M, Naredi S, Koshkinen LO. Effective ICP Reduction by Decompressive Craniectomy in Patients with Severe Traumatic Brain Injury. Treated by an ICP-Targeted Therapy. J Neurotrauma 2007;24:927-35.

2. Howard JL, Cipolle MD, Anderson M, Sabella V, Shollenberger D, Li PM, et al. Outcome after decompressive craniectomy for the treatment of severe traumatic brain injury. J Trauma 2008;65:380-5.

3. Bor-Seng-Shu E, Paiva WS, Figueiredo EG, Fujimoto Y, de Andrade AF, Fonoff ET, et al. Posttraumatic refractory intracranial hypertension and brain herniation syndrome: Cerebral hemodynamic assessment before decompressive craniectomy. Biomed Res Int 2013;2013:750809.

4. Bor-Seng-Shu E, Figueiredo EG, Fonoff ET, Fujimoto Y, Panerai RB, Teixeira MJ. Decompressive craniectomy and head injury: Brain morphometry, ICP, cerebralhemodynamics, cerebral microvascular reactivity, and neurochemistry. Neurosurg Rev 2013;36:361-70.

5. Bor-Seng-Shu E, Figueiredo EG, Amorim RL, Teixeira MJ, Valbuza JS, de Oliveira MM, et al. Decompressive craniectomy: A meta-analysis of influences on intracranial pressure and cerebral perfusion pressure in the treatment of traumatic brain injury. J Neurosurg 2012;117:589-96.

6. Amorim RL, Bor-Seng-Shu E, Gattás GS, Paiva W, de Andrade AF, Teixeira MJ. Decompressive craniectomy and cerebral blood flow regulation in head injured patients: A case studied by perfusion CT. J Neuroradiol 2012;39:346-9.

7. AarabiB,HesdorfferDC, SimardJM, Ahn ES, Aresco C, Eisenberg HM, etal. Comparative study of decompressive craniectomy after mass lesion evacuation in severe head injury. Neurosurgery 2009;64:927-39.

8. Polin RS, Shaffrey ME, Bogaev CA, Tisdale N, Germanson D, Bocchicchio B, et al. Decompressive bifrontal craniectomyin the treatment of severe refractory posttraumatic cerebral edema. Neurosurgery 1997;41:84-92.

9. Schmidt JH, Reyes BJ, Fischer R Flaherty SK. Use of hinge craniotomy for cerebral decompression. Technical note. J Neurosurg 2007;107:678-82.

10. Mitchell P, Tseng M, Mendelow AD. Decompressive craniectomy with latticeduraplasty. Acta Neurochir (Wien) 2004;146:159-60.

11. Csokay A, Egyud L, Nagy L, Pataki G. Vascular tunnel creation to improve the efficacy of decompressive craniotomy in post-traumatic cerebral edema and is chemic stroke. Surg Neurol 2002;57:126-9. 
12. De Bonis P, Pompucchi A, Mangiola A, Rigante L, Anile C. Post-traumatic hydrocephalus after decompressive craniectomy: An underestimated risk factor. J Neurotrauma 2010;27:1965-70.

13. Munch E, Horn P, Schurer L, Piepgras A, Paul T, Schmiedek P. Management of severe traumatic brain injury by decompressive craniectomy. Neurosurgery 2000;47:315-22.

14. Honeybul S, Ho KM. Long-term complications of decompressive craniectomy for head injury. J Neurotrauma 2011;28:929-35.

15. Wirtz CR, Steiner T, Aschoff A, Schwab S, Schnippering H, Steiner H, et al. Hemicraniectomy with dural augmentation in medically uncontrollable hemispheric infarction. Neurosurg Focus 1997;2:E3.

16. Wagner S, Schnippering H, Aschoff A, Koziol JA, Schwab S, Steiner T. Suboptimum hemicraniectomy as a cause of additional cerebral lesions in patients with malignant infarction of the middle cerebral artery. J Neurosurg 2001;94:693-6.

17. Jiang JY, Xu W, Li WP, Xu WH, Zhang J, Bao YH, et al. Efficacy of standard trauma craniectomy for refractory intracranial hypertension with severe traumatic brain injury: A multicenter, prospective, randomized controlled study. J Neurotrauma 2005;22:623-8.

18. Qiu W, Guo C, Shen H, Chen K, Wen L, Huang H, et al. Effects of unilateral decompressive craniectomy on patients with unilateral acute post-traumatic brain swelling after severe traumatic brain injury. Crit Care 2009;13:R185.
19. Skoglund TS, Eriksson-Ritzen C, Jensen C, Rydenhag B. Aspects on decompressive craniectomy in patients with traumatic head injuries. J Neurotrauma 2006;23:1502-9.

20. Gao CP, Ang BT. Biomechanical modeling of decompressive craniectomy in traumatic brain injury. Acta Neurochir Suppl 2008;102:279-82.

21. Honeybul S. Complications of decompressive craniectomy for head injury. J Clin Neurosci 2010;17:430-5.

22. Cooper DJ, Rosenfeld JV, Murray L, Arabi YM, Davies AR, D’urso P, et al. Decompressive Craniectomy in diffuse traumatic brain injury. $\mathrm{N}$ Engl J Med 2011;364:1493-502.

23. Servadei F. Clinical value of decompressive craniectomy. N Engl J Med 2011;364:1558-9.

How to cite this article: Sedney CL, Julien T, Manon J, Wilson A. The effect of craniectomy size on mortality, outcome, and complications after decompressive craniectomy at a rural trauma center. J Neurosci Rural Pract 2014;5:212-7.

Source of Support: Nil. Conflict of Interest: Sedney: Nothing to disclose; Julien: Consultant for Stryker spine, Orthovita, Globus Medical, Biomet. Lecturer for Stryker Spine, Globus Medical. Stock holder for Orthovita; Manon: Nothing to disclose; Wilson: Nothing to disclose.

Announcement

\section{Android App}

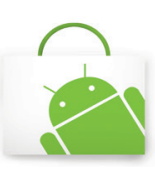

A free application to browse and search the journal's content is now available for Android based mobiles and devices. The application provides "Table of Contents" of the latest issues, which are stored on the device for future offline browsing. Internet connection is required to access the back issues and search facility. The application is compatible with all the versions of Android. The application can be downloaded from https://market.android.com/details?id=comm.app.medknow. For suggestions and comments do write back to us. 